\title{
Fungal Profile and Its Characteristics in Patients of Otomycosis-A Prospective Study
}

SUSHIL KUMAR AGGARWAL'1, KIRTI JAISWAL²

\section{ABSTRACT}

Introduction: Fungal ear infection is one of the most common infections, which is termed as otomycosis. Common causative agents are Aspergillus and Candida species.

Aim: To find the prevalence, identification and susceptibility testing of fungal species in samples collected from patients with ear infection.

Materials and Methods: Samples were collected from 105 patients of otomycosis and were inoculated on SDA and $\mathrm{BA}$ tubes. $\mathrm{KOH}$ preparations were made to see the presence of fungal elements. Morphology of filamentous fungi was identified by using Lacto Phenol Cotton Blue (LPCB) and yeast-like fungi by Gram stain and CHROM agar medium. Susceptibility testing was done to find out the sensitivity and resistivity against Voriconazole, Itraconazole, Fluconazole and Amphotericin-B by disc diffusion method.

Results: Otomycosis was common in the age group between 2040 years, and males were more affected as compared to females. Common isolates found were moulds such as Aspergillus niger (14.89\%), A. flavus (21.2\%), A. fumigatus (21.2\%), Rhizopus oryzae $(2.1 \%)$ and Scedosporium (2.1\%); yeast-like fungi were Candida tropicalis (34\%) and C. glabrata (8.5\%).

Conclusion: Microscopic examination and fungal culture is essential for confirmation of the diagnosis. Proper identification of fungus followed by antifungal treatment according to susceptibility testing is important for treating such patients.

Keywords: Antifungal susceptibility testing, Aspergillus, Candida

\section{INTRODUCTION}

Ear is continuously exposed to biotic elements of the atmosphere, as a consequence easily accessible to various microorganisms, including fungi which leads to inflammation and fluid development inside the ear. Local lesions observed in otitis externa, such as congestion, increases the vascular permeability and temperature to create favourable conditions for the growth of fungi and development of mycoses both in the external and middle ear [1]. Fungi are abundant in soil or sand, which contains decomposing vegetable matter. These are dehydrated rapidly in tropical area and blown in wind as small dust particles. The air borne fungal spores are carried by water vapours, which correlates with higher rate of infection, when relative humidity rises to $80 \%$ [2]. Andrall and Gaverret were the first to describe fungal ear infection in 1843 and by Mayer in 1844 [3].

Otomycosis has been described as superficial fungal infection of the external auditory canal while infections in middle ear occur due to perforated tympanic membrane. Permanent damage of the middle ear and inner ear is caused by chronic fungal infection of ear [4]. It is characterised by the presence of otalgia, ear discharge, pruritus, ear fullness, hearing loss. Common predisposing factors are heat and humidity, change in $\mathrm{pH}$, temperature, qualitative and quantitative change in cerumen, immuno-compromised patients and systemic diseases, trauma, Chronic Suppurative Otitis Media (CSOM), use of antibiotics and non-sterile oils [4-6].

Common fungal isolates are Aspergillus and Candida species. In recent years, there has been an increase in the incidence of otomycosis due to increase in prevalence of co-morbid conditions like diabetes mellitus, malignancies and use of ear buds $[4,5,7]$. Although there has been controversy with respect to whether the fungi are the true infective agents versus mere colonisation of the species as a result of compromised local host immunity secondary to bacterial infection, most clinical and laboratory evidence show that otomycosis is a true pathological entity [2]. Otomycosis is sporadic and is caused by a wide variety of fungi, most of which are saprophytes growing in diverse types of environmental conditions [3]. The fungus is usually a secondary contaminant in cases of otitis externa and hence can be found mixed with bacterial infections also [8]. Though the disease can be diagnosed clinically, microscopic examination and fungal culture is required for confirmation of the diagnosis. Antifungal susceptibility testing methods help to detect the antifungal resistance. Diagnosis and management of otomycosis can be challenging in immunocompromised patients; also recurrences are more common among them.

This research on otomycosis described clinical presentation, fungal profile and its characteristics along with antifungal susceptibility testing of various commonly involved species and whether the fungal species found in the study were similar to other studies mentioned in literature. We also studied in detail about the regional differences in fungal species causing otomycosis.

\section{MATERIALS AND METHODS}

\section{Study Group}

Mycological analysis was carried out on debris or exudate samples from the ear auditory canals of 105 patients clinically diagnosed to have otomycosis infection, collected from Outpatient Department (OPD) of Otorhinolaryngology of Sir Sunder Lal Hospital, Banaras Hindu University, Varanasi, Uttar Pradesh, India, from January 2018 to December 2018. All patients were informed about the study and consent was taken before taking the ear swab. The collection of ear swab and to send it for culture and sensitivity, is a routine procedure at the study institute. However, all the ethical considerations were taken care of.

\section{Ethical Considerations}

All patients were informed about the study and proper consent from all the patients was taken before taking the ear swab. Hence, no ethical obligations were involved in our study.

\section{Collection of Samples}

The samples were collected under aseptic conditions using sterile cotton swab from the external auditory canal. To diagnose otomycosis, detailed history, clinical examination, otoscopic findings and laboratory identification of fungus were considered [9]. 


\section{Culture}

Two samples were taken, one was used for inoculation on SDA and BA tubes and other was used for $\mathrm{KOH}$ mount. The inoculated SDA tubes were incubated on $25^{\circ} \mathrm{C}$ and examined after 2-3 days of incubation. BA tubes were incubated on $37^{\circ} \mathrm{C}$ for 24 hours.

\section{Identification}

Direct microscopy: For detection of fungal elements, $\mathrm{KOH}(10 \%)$ preparation was used. Gram stain was done for identification for yeast-like fungi and LPCB was used to identify the filamentous fungi. CHROM Agar was used to identify the Candida species.

\section{Antifungal Susceptibility Testing}

Candida species: The disc diffusion assay was performed according to CLSI guideline (M44-A2) to determine the susceptibility of obtained isolates. In brief, Mueller-Hinton agar was supplemented with $2 \%$ glucose, provided a suitable growth for most of the yeasts, and $0.5 \mathrm{mg} / \mathrm{L}$ methylene blue was used. The $\mathrm{pH}$ of the medium was maintained between 7.2 and 7.4 after gelling and the agar was $4 \mathrm{~cm}$ high. Inoculum was taken with a sterile swab stick from sub-cultured SDA tubes, mixed with normal saline (1 $\mathrm{mL})$ and prepared in tubes, inoculum was standardised to $0.5 \mathrm{McF}$ arland, it was swabbed on MHA plates supplemented with $2 \%$ glucose and $0.5 \%$ methylene blue, antifungal drugs (Voriconazole, Itraconazole, Fluconazole, Amphotericin-B) were applied and were incubated at $35^{\circ} \mathrm{C}$ for 24 hours; some strains showed insufficient growth and these strains required $48 \mathrm{hrs}$ of incubation to grow.

\section{Filamentous Fungi}

CLSI guideline (M-51A) protocol was followed. PDA sub-cultured tubes were taken, 1 drop of Tween 80 was added in each of the tubes, $1 \mathrm{~mL}$ normal saline was added and mixed with the spores. Then, 1-2 drops of prepared solution was taken in tubes of prepared normal saline, which was standardised to $0.1 \mathrm{McF}$ arland. The prepared mixture was then swabbed on plain MHA plates with the swab stick, and antifungal drugs (VIFA) were applied and incubated at $25^{\circ} \mathrm{C}$ for 48 hours; some strains showed insufficient growth and would require 72 hours of incubation.

\section{RESULTS}

A total of 105 patients with suspected otomycosis were included in the study; 114 samples were obtained (as some patients had otomycosis in both the ears).

The most prevalent age-group was 20-40 years (44 patients) followed by age-group of 1-20 years [Table/Fig-1].

\begin{tabular}{|l|c|c|c|}
\hline Age in years & Male & Female & Total \\
\hline $1-20$ & 17 & 16 & 33 \\
\hline $20-40$ & 26 & 18 & 44 \\
\hline $40-60$ & 11 & 06 & 17 \\
\hline $60-80$ & 08 & 02 & 10 \\
\hline $80-100$ & 01 & 00 & 01 \\
\hline Total & 63 & 42 & 105 \\
\hline
\end{tabular}

The common presenting symptoms solely or in combination encountered in the study group have been summarised in [Table/Fig-2]. Ear discharge (72.38\%) was the most common complaint.

Otomycosis was unilateral in 105 (92\%) cases and 9 (7.8\%) cases were bilateral. Among the unilateral cases, the right side (48.2\%) showed predominance [Table/Fig-3].

Majority (15.2\%) of the patients gave history of CSOM [Table/Fig-4]. [Table/Fig-5] shows the list of associated medical conditions in the study, out of which a majority of $7.6 \%$ had common cold and cough.
Also, it was found that 6 patients with immuno-compromised state and 35 patients with immuno-competent state were positive for otomycosis. Three patients were bilaterally positive in immunocompetent patients while rest of the patients were unilaterally positive.

\begin{tabular}{|l|c|c|}
\hline Symptoms & No. of patients & Percentage (\%) \\
\hline Pruritus & 28 & 26.6 \\
\hline Ear discharge & 76 & 72.38 \\
\hline Tinnitus & 50 & 47.6 \\
\hline History of hearing loss & 43 & 40.9 \\
\hline Ear pain & 18 & 17.1 \\
\hline Ear fullness & 10 & 9.5 \\
\hline
\end{tabular}

\begin{tabular}{|l|c|c|}
\hline Ear & No. of patients & Percentage (\%) \\
\hline Right & 55 & 48.2 \\
\hline Left & 50 & 43.8 \\
\hline Bilateral & 9 & 7.89 \\
\hline
\end{tabular}

\begin{tabular}{|l|c|c|}
\hline Factors & No. of patients & Percentage (\%) \\
\hline Use of oils, antibiotics and self-cleaning & 6 & 5.71 \\
\hline Trauma & 4 & 3.8 \\
\hline Swimming & 1 & 0.95 \\
\hline CSOM & 16 & 15.2 \\
\hline Presence of ear wax & 2 & 1.9 \\
\hline
\end{tabular}

\begin{tabular}{|l|c|c|}
\hline Medical history & No. of patients & Percentage (\%) \\
\hline Diabetes & 03 & 2.85 \\
\hline Blood pressure & 02 & 1.9 \\
\hline Cold and cough & 08 & 7.6 \\
\hline Heart problem & 01 & 0.95 \\
\hline Herpes & 01 & 0.95 \\
\hline Immuno-compromised & 06 & 5.7 \\
\hline Immuno-competent & 35 & 33.3 \\
\hline \multicolumn{2}{|l|}{ [Table/Fig-5]: Associated medical history. }
\end{tabular}

Only 45 samples were positive for the presence of fungal elements. Out of 114 samples, 64 were negative both by microscopy and culture, two were positive with $\mathrm{KOH}$ but no growth on culture and 3 were negative with $\mathrm{KOH}$ but no growth [Table/Fig-6].

\begin{tabular}{|l|c|c|c|c|}
\hline Positivity & Culture positive & Culture negative & No growth & Total \\
\hline $\mathrm{KOH}$ positive & 22 & 2 & 02 & 26 \\
\hline $\mathrm{KOH}$ negative & 21 & 62 & 03 & 88 \\
\hline Total & 45 & 64 & 05 & 114 \\
\hline
\end{tabular}

A total of 45 out of 114 samples, were positive for fungal growth by culture. The most common fungal isolates belonged to the species of Aspergillus. Aspergillus flavus and fumigatus (20\%) were the most common isolate followed by Aspergillus niger (15.5\%) and Aspergillus terreus (4.4\%). Two samples were moulds and two samples having yeast like species (which could not be identified) [Table/Fig-7].

Aspergillus niger was found in 7 samples, all were sensitive to Voriconazole showing a clear zone inhibition. Yeast-like fungi included 10 samples of Candida tropicalis, all were sensitive to Voriconazole showing a clear zone of inhibition. All 10 were sensitive to Fluconazole and Amphotericin-B. Candida glabrata was grown in 2 samples; all were sensitive to Voriconazole, Itraconazole, Fluconazole and Amphotericin-B [Table/Fig-8]. 


\begin{tabular}{|l|c|c|}
\hline Fungal species & No. of patients & Percentage (\%) \\
\hline Aspergillus niger & 07 & 15.5 \\
\hline Aspergillus flavus & 9 & 20 \\
\hline Aspergillus fumigatus & 9 & 20 \\
\hline Aspergillus terreus & 02 & 4.4 \\
\hline Rhizopus oryzae & 01 & 2.2 \\
\hline Scedosporium & 01 & 2.2 \\
\hline Candida tropicalis & 10 & 22.2 \\
\hline Candida glabrata & 02 & 4.4 \\
\hline Species unidentified (molds) & 02 & 4.4 \\
\hline Species unidentified (yeast-like) & 02 & 4.4 \\
\hline Total & 45 & 100 \\
\hline Trable/Fig-7]: Spectrum of fungal isolates from otomycosis patients (Total samples-45).
\end{tabular}

\begin{tabular}{|c|c|c|c|c|c|c|c|c|c|c|c|c|}
\hline \multirow[t]{2}{*}{$\begin{array}{l}\text { Fungal } \\
\text { species }\end{array}$} & \multicolumn{3}{|c|}{ Voriconazole } & \multicolumn{3}{|c|}{ Itraconazole } & \multicolumn{3}{|c|}{ Fluconazole } & \multicolumn{3}{|c|}{ Amphotericin-B } \\
\hline & $S$ & 1 & $R$ & $S$ & 1 & $R$ & $S$ & I & $\mathrm{R}$ & $S$ & 1 & $\mathrm{R}$ \\
\hline A.niger & 07 & 00 & 00 & 03 & 02 & 02 & 01 & 01 & 05 & 07 & 00 & 00 \\
\hline A.flavus & 08 & 00 & 01 & 06 & 03 & 00 & 01 & 01 & 07 & 02 & 01 & 06 \\
\hline A.terreus & 02 & 00 & 00 & 00 & 02 & 00 & 00 & 00 & 02 & 00 & 00 & 02 \\
\hline C.tropicalis & 10 & 00 & 00 & 08 & 00 & 02 & 10 & 00 & 00 & 10 & 00 & 00 \\
\hline C.glabrata & 02 & 00 & 00 & 02 & 00 & 00 & 02 & 00 & 00 & 02 & 00 & 00 \\
\hline
\end{tabular}

\section{DISCUSSION}

In the present study, analysis of the age-group revealed that otomycosis can affect any age from 1 to 100 years. However, the incidence was highest in the age-group of 20-40 years, similar to the findings mentioned by Fasunla J et al., and Ologe FE et al., [10,11]. The higher incidence in these age-groups was due to the fact that these people are more exposed to the mycelia due to occupational exposure, travelling etc., whereas the older and younger age-groups are less exposed to these pathogens.

In the study, males were more commonly affected than females, as males spend more time outdoors leading to more exposure to fungal spores. It is well known that the outdoor air is an important vector for locally prevalent fungal flora. Our study correlates well with the study of Than $\mathrm{KM}$ et al., Kaur R et al., and Ho T et al., which showed $58 \%$, $60 \%$ and $56 \%$ incidence in males, respectively [12-14]. Unilateral involvement was seen in $92 \%$ in our study, which correlated with the study done by Paulose $\mathrm{KO}$ et al., in which unilateral involvement was $87 \%$ [15]. Ho T et al., observed a bilateral involvement in $7 \%$ of the patients, which corresponds to the present study where we found bilateral otomycosis in 7.89\% patients [14].

Common symptoms of otomycosis as mentioned in literature are itching, ear discharge, ear pain, blocking sensation, decreased hearing and tinnitus $[16,17]$. In our study, ear discharge $(34 \%)$ was the more common symptom followed by tinnitus (22\%).

In the present study, immunocompromised patients having otomycosis were commonly associated with co-morbid illnesses like common cold \& cough, diabetes, high blood pressure, heart problems and herpes. Also, 3.8\% of the patients had trauma with stick, feather, metal picker, pin etc., The habit of cleaning the ear with feathers, match-stick or contaminated finger tips is known to encourage the inoculation and growth of the spores of fungus on the moist external auditory canal especially in patients applying oil to the area. Excessive cerumen in some patients with poor personal hygiene favours the germination of spores and conidia of the prevalent fungi.

The moisture, warmth, and acidic $\mathrm{pH}$ of the external auditory canal provide ideal growth medium for the fungi. Swimming was also a predisposing factor in our study, similar to the study by Ozcan KM et al., Fasunla $\mathrm{J}$ et al., and Paulose $\mathrm{KO}$ et al., $[5,10,15]$.
Lack of formal education in people in many parts of India has let them to believe in myths that coconut oil application in ears is beneficial for a variety of ear ailments. Coconut oil has been reported to be sporostatic and therefore may help preserve the viability of fungal conidia deposited in the external ear for long and indirectly contribute to occurrence of otomycosis [18]. Similarly the use of mustard oil is associated with high incidence of otomycosis [17].

Overview of literature shows that among the fungal isolates, Aspergillus niger and Candida were the most common species causing otomycosis worldwide but more than 50 causative fungi species have been isolated in their studies by various authors which belong to genera Penicillium, Fusarium, Mucoraceae, Scopulariopsis, Alternaria, Malassezia and as well various dermatophytes [16,19]. In the present study, we recorded $60 \%$ of Aspergillus species, which correlated with the study of Satish HS et al., (54\%) and Paulose KO et al., $(54.4 \%)[3,15]$. However, present study differed from the study conducted by Aneja KR et al., as the most prevalent microorganism in their study was Aspergillus niger (43.31\%), whereas most prevalent species in present study was Candida tropicalis (22.2\%) [7]. In some other studies mentioned in the literature, yeast fungi such as $C$. albicans had more frequency than $A$. niger, similar to this study [19-22].

Aspergillus flavus species found in this study was 20\%, which correlated well with the study of Satish HS et al., and Yahiya MM et al., [3,20]. Infection with Candida can be more difficult to detect clinically because of its lack of a characteristic appearance like Aspergillus and can present as otorrhea not responding to aural antimicrobials. Otomycosis attributed to Candida is often identified by culture data $[13,15]$. The colour of discharge from the ear and also the laboratory identification may help towards the probable identification of the disease agent [22]. Usually the black discharge may be due to Aspergillus genus, especially A. niger, and creamy or white discharge may be due to Candida genus [23].

Some rarest species had also been found in the index study, with 2 isolates of $A$. terreus, 1 isolate of Rhizopus oryzae and 1 isolate of Scedosporium. The Scedosporium genus encompasses a group of filamentous fungi isolated from water, soil, stalled or polluted water all over the world. Two species cause human infection: S. apiospermum (asexual anamorphous of Pseudoallescheria boydii) and S. prolificans (S. inflatum). These are considered rare human pathogens, especially in immunocompromised patients $[24,25]$. Next common group in the study was Candida species, accounting for $(26.6 \%)$ of total isolates. This correlated with the study by Satish HS et al., (53.4\%) and Viswanatha B et al., [3,26]. There was a difference in isolation of fungi in the present study when compared to other studies which may be due to geographical variation [Table/Fig-9] [7,10,13,17-19,22,26,27].

There is no consensus on the most effective agent. Application of appropriate topical antifungal agents coupled with frequent mechanical debridements usually results in prompt resolution of symptoms, though recurrence or residual disease can occur [28]. Administration of mildly acidic drops, such as boric acid and alcohol, or modified Burow's solution can be a cost-effective therapy for primary treatment of otomycosis [29]. Clotrimazole and Fluconazole are very effective drugs in the treatment of otomycosis [30].

In the index study, susceptibility test disc diffusion method was performed against Voriconazole, Itraconazole, Fluconazole and Amphotericin-B. It was found that patients suffering from Aspergillus niger infection were sensitive to Voriconazole and Amphotericin- B, showing a clear zone of inhibition. Aspergillus flavus and terreus were also sensitive to Voriconazole. Both of them were resistant to Fluconazole and Amphotericin-B. Yeast-like fungi included Candida tropicalis were sensitive to Voriconazole, Itraconazole, Fluconazole and Amphotericin-B. Candida glabrata was sensitive 


\begin{tabular}{|l|c|c|c|c|c|c|c|}
\hline Authors & Year & A. niger/A. fumigatus/A. flavus & Candida sp. & Mucor & Rhizopus & Scedosporium & Species unidentified \\
\hline Kaur R et al., [13] & 2000 & $36.9 / 41.1 / 1.4$ & 13.7 & 1.4 & 2.7 & - & 1.4 \\
\hline Pradhan B et al., [17] & 2003 & $25.5 / 6.6 / 37.7$ & 10.4 & - & - & - & - \\
\hline Vishwanatha B et al., [26] & 2012 & $56 / 18 /-$ & 16 & - & - & - \\
\hline Pontis ZBVDS et al., [19] (Brazil) & 2009 & $20 / 5 / 10$ & 55 & - & - & - & - \\
\hline Aneja KR et al., [7] & 2010 & $39.8 / 12.9 / 16.6$ & 10.2 & - & - & - & - \\
\hline Fasunla J et al., [10] (Nigeria) & 2008 & $48.35 / 33.96 / 5.43$ & 12.26 & - & - & - & - \\
\hline Barati B et al., [27] (Iran) & 2011 & $41.6 / 5.5 / 49$ & 7.6 & - & - & - & - \\
\hline Jain SK and Agarwal SC [18] & 1992 & $56.3 / 15.6 / 4.7$ & 6.3 & 6.3 & - & - & - \\
\hline Jaiswal SK [22] & 1990 & $34 /-/-$ & 46 & - & 12 & - & 3 \\
\hline Present study & 2019 & $15.5 / 20 / 20$ & 22.2 & - & 2.2 & 2.2 & 8 \\
\hline
\end{tabular}

to Voriconazole, Itraconazole, Fluconazole and Amphotericin-B. Overall, it was concluded that all the fungal species were sensitive to Voriconazole and Amphotericin-B, though some were resistant to Fluconazole and Itraconazole.

\section{LIMITATION}

1. Ear swab method was used for sample collection, which is not a good method for diagnosis of species in otomycosis.

2. A control group should have been taken, in which sample from patients with purulent ear discharge should have been examined for diagnosis of species, so that the various pathogens involved in ear discharge could have been compared.

\section{CONCLUSION}

The most common clinical feature was ear discharge and the common age-group was 20-40 years. CSOM was the most common predisposing factor. The most common fungal species found was C. tropicalis. All species were sensitive to both Voriconazole and Amphotericin-B.

\section{REFERENCES}

[1] Kurnatowski P, Filipiak A. Otomycosis: Prevalence, clinical symptoms, therapeutic procedure. Mycoses. 2001;44(11):472-79.

[2] Viswanatha B, Naseeruddin K. Fungal infections of the ear in immunocompromisedhost. Mediterranean Journal of Hematology and Infectious Diseases. 2011;3:01-04.

[3] Satish HS, Viswanatha B, Manjuladevi M. A clinical study of otomycosis. Journal of Dental Medical Science. 2013;5:57-62.

[4] Gokale SK, Suligavi SS, Baragundi M, Anushka D, Manjula R. Otomycosis: Aclinico mycological study. International Journal of Medical and Health Sciences. 2013;2:218-23.

[5] Ozcan KM, Ozcan M, Karaarslan A, Karaarslan F. Otomycosis in Turkey: Predisposing factors, etiology and therapy. J Laryngol \& Otology. 2003;117:39-42.

[6] Jackman A, Ward R, April M, Bent J. Topical antibiotic induced otomycosis. Int J of Pediatric Otorhinolaryngology. 2005;69(6):857-60

[7] Aneja KR, Sharma C, Joshi R. Fungal infection of the ear: A common problem in the north eastern part of Haryana. International Journal of Pediatric Otorhinolaryngology. 2010;74(6):604-07.

[8] Kazemi A, Majidinia M, Jaafari A, Mousavi SA, Mahmoudabadi AZ, Alikhah $\mathrm{H}$. Etiologic agents of otomycosis in the North-Western area of Iran. Jundishapur Journal of Microbiology. 2015;8(9):e21776.

[9] Vennewald I, Klemm E. Otomycosis: Diagnosis and treatment. Clin Dermatol. 2010;28(2):202-11
[10] Fasunla J, Ibekwe T, Onakoya P. Otomycosis in western Nigeria. Mycoses. 2008:51(1):67-70.

[11] Ologe FE, Nwabuisi C. Treatment outcome of otomycosis in llorin, Nigeria. West African Journal of Medicine. 2002;21(1):34-36.

[12] Than KM, Naing KS, Min M. Otomycosis in Burma and its treatment. Am J Trop Med Hyg. 1980;29(4):620-23.

[13] Kaur R, Mittal N, Kakkar M, Aggarwal AK, Mathur MD. Otomycosis: A clinicomycologic study. Ear, Nose \& Throat Journal. 2000;79(8):606.

[14] Ho T, Vrabec JT, Yoo D, Coker NJ. Otomycosis: clinical features and treatment implications. Otolaryngol Head Neck Surg. 2006;135(2):787-91.

[15] Paulose KO, Al Khalifa S, Shenoy P, Sharma RK. Mycotic infection of the ear (Otomycosis): a prospective study. J Laryngol Otol. 1989;103:30-35.

[16] Prasad SC, Kotigadde S, Shekhar M, Thada ND, Prabhu P, D'Souza T, et al. Primary otomycosis in the Indian subcontinent: Predisposing factors, microbiology \& classification. International Journal of Microbiology. 2014;2014:636493.

[17] Pradhan B, Tuladhar RN, Amatya MR. Prevalence of Otomycosis in outpatient department of otolaryngology in Tribhuvan University Teaching Hospital, Kathmandu, Nepal. Annals of Otology, Rhinology and Laryngology. 2003;112(4):384-87.

[18] Jain SK, Aggarwal SC. Sporostatic effect of some oils against fungi causing Otomycosis. Indian Journal of Medical Sciences. 1992;46(1):1-6.

[19] Pontes ZBVDS, Silva ADF, Lima EDO, de Holanda Guerra M, Oliveira NMC, Carvalho MFFP, et al. Otomycosis: A retrospective study. Brazilian Journal of Otorhinolaryngology. 2009;75(3):367-70.

[20] Yahiya MM, Al-Habib HM, Shehab MN. Otomycosis: A common problem in North Iraq. Journal of Laryngology and Otology. 1990;104(5):387-89.

[21] Ninkovic G, Dullo V, Saunders NC. Microbiology of otitis externa in the secondary care in United Kingdom and antimicrobial sensitivity. Auris Nasus Larynx. 2008;35(4):480-84.

[22] Jaiswal SK. Fungal infection of ear and its sensitivity pattern. Indian Journal of Otolaryngology. 1990;42(1):19-22.

[23] Hirsch BE. Infections of the external ear. Am J Otolaryngol. 1992;13(3):145-55.

[24] Milne L JR, Mckerrow WS, Paterson WD. Pseudallescheriasis in northern Britain. J Med Vet Mycol. 1986;24(5):377-82.

[25] Wilson CM, O'Rourke EJ, McGinnis MR. Scedosporium inflatum: clinical spectrum of a newly recognized pathogen. J Infect Dis. 1990;161:102-07.

[26] Viswanatha B, Sumatha D, Vijayashree MS. Otomycosis in immunocompetent and immunocompromised patients; comparative study and literature review. ENT Journal. 2012;91(3):114-21.

27] Barati B, Okhovvat SAR, Goljanian A, Omrani MR. Otomycosis in centra Iran: A clinical and mycological study. Iranian Red Crescent Medical Journal. 2011;13(12):873-76

[28] Stern JC, Shah MK, Lucente FE. In vitro effectiveness of 13 agents in otomycosis and review of literature. Laryngoscope. 1988;98:1173-77.

[29] Manning SC. Mycoses. In: Paparella MM, ShumrickDA, Gluckman JL, Meyerhof WL, eds. Otolaryngology, $3^{\text {rd }}$ Edn. Philadelphia: WB Saunders, 1991:589-96.

[30] Yadav SPS, Gulia JS, Jagat S, Goel AK. Role of ototopical Fluconazole and Clotrimazole in management of Otomycosis. Indian Journal of Otology. 2007;13;12-15.

\section{PARTICULARS OF CONTRIBUTORS:}

1. Assistant Professor, Department of ENT, Institute of Medical Sciences, Banaras Hindu University, Varanasi, Uttar Pradesh, India

2. Postgraduate, Department of Microbiology, Institute of Medical Sciences, Banaras Hindu University, Varanasi, Uttar Pradesh, India.

NAME, ADDRESS, E-MAIL ID OF THE CORRESPONDING AUTHOR:

Dr. Sushil Kumar Aggarwal,

Banaras Hindu University, Varanasi-221005, Uttar Pradesh, India.

E-mail: doc.sushil.pgi@gmail.com

Date of Submission: Jul 27, 2019 Date of Peer Review: Jul 31, 2019 Date of Acceptance: Aug 02, 2019

FINANCIAL OR OTHER COMPETING INTERESTS: None.
Date of Publishing: Oct 01, 2019 